\title{
Oxidative stress in bicuspid aortic valve-related aortopathy: Hand-me-downs and yoga pants
}

\author{
Jeffrey A. Jones, $\mathrm{PhD}$
}

From Cardiothoracic Surgery Research, Department of Surgery, Medical University of South Carolina, and the Research Service, Ralph H. Johnson Veterans Affairs Medical Center, Charleston, SC.

Disclosures: Author has nothing to disclose with regard to commercial support.

Received for publication June 7, 2017; accepted for publication June 12, 2017; available ahead of print July 8, 2017.

Address for reprints: Jeffrey A. Jones, PhD, Division of Cardiothoracic Surgery Research, Medical University of South Carolina, Strom Thurmond Research Building, 114 Doughty St, Suite 338, MSC778, Charleston, SC 29425 (E-mail: jonesja@musc.edu).

J Thorac Cardiovasc Surg 2017;154:1764-5

0022-5223/\$0.00

Published by Elsevier Inc. on behalf of The American Association for Thoracic Surgery http://dx.doi.org/10.1016/j.jtcvs.2017.06.025

In this issue of the Journal, Billaud and colleagues ${ }^{1}$ present compelling evidence that ascending aortic tissue from patients with bicuspid aortic valve (BAV) shows elevated oxidative damage with an elevated abundance of reactive oxygen species (ROS). Since the initial description of the BAV by Leonardo da Vinci and its subsequent clinical association with aortopathy by Abbott in $1927,,^{2}$ there has been great debate centered on the underlying mechanisms contributing to the etiology of BAV-related aortic disease. Specifically, 2 primary camps have emerged, one focused on identifying underlying genetic mutations that contribute to altered aortic remodeling and dilatation and the other concentrating on the role of excessive mechanical stress generated by aberrant blood flow redirected by fused valve leaflets impinging on the aortic wall. ${ }^{3}$ Although definitive causality has yet to be defined by either underlying mechanism, data slowly have accumulated that support both etiologies.

In the present manuscript, the authors quantified elevated superoxide-anion levels in ascending aortic tissue from patients with BAV without aneurysm compared with tissue from patients with BAV with aneurysm, or patients with a normal trileaflet aortic valve in the presence or absence of disease. Importantly, BAV tissues failed to show elevated superoxide-dismutase activity, a protective enzyme that functions to scavenge superoxide-anions and convert them to the less-reactive intermediate, hydrogen peroxide. There was a noted increase in peroxidase activity, capable of clearing the hydrogen peroxide; however, the investigators also identified increased oxidative damage, as measured by an increase in 8-iso-prostaglandin-F2-alpha, in all BAV aortic specimens compared with aortic specimens from patients with trileaflet aortic valve. These results suggested that the antioxidant defense was insufficient to completely abrogate the effects of the increased ROS. The authors contend that these changes in oxidative stress may contribute to alterations in the integrity of the extracellular matrix, which in turn may be associated with BAV-related aortopathy.

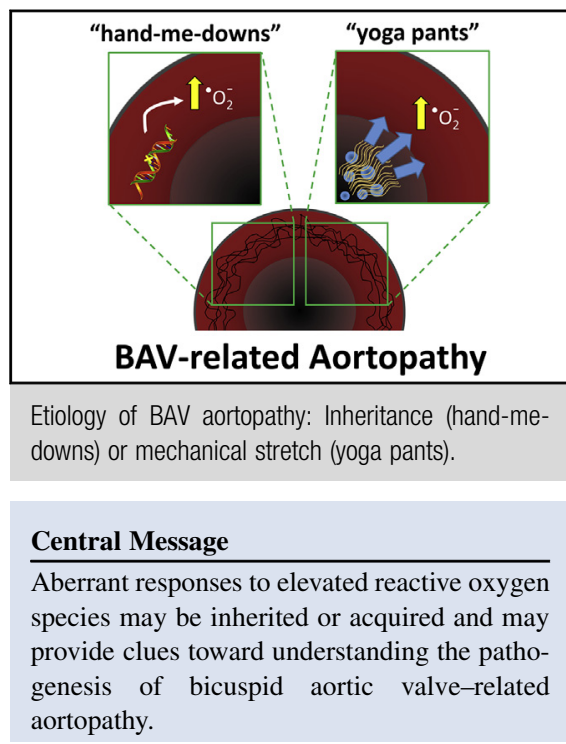

See Article page 1756.

There are several pathways and mediators that contribute to ROS production in medial aortic cells, including nicotinamide adenine dinucleotide phosphate oxidase, xanthine oxidase, uncoupled endothelial nitric oxide synthase, and electron leak from the mitochondrial respiratory chain. These are balanced by multiple mediators designed to clear ROS, including superoxide-dismutase, catalase, and various peroxidases. Although altered expression of many of these proteins has been associated with changes in myocardial protection in ischemic heart disease, to date there have been no reports of underlying genetic mutations in the genes of these mediators associated with BAV-related aortopathy. Interestingly, however, changes in these mediators resulting in elevated ROS production have been associated with hypertension and cyclic mechanical stretch. ${ }^{4}$ This may suggest that the altered antioxidant defenses observed by Billaud and colleagues may be a consequence of elevated wall stress secondary to BAV-induced alterations in blood flow over the valve. Studies by this same group of investigators previously have identified aortic regional differences in antioxidant gene expression in patients with BAV. ${ }^{5}$ Moreover, studies by Della Corte and Cotrufo have shown changes in regional extracellular matrix protein production and deposition in the convex versus concave aspect of the ascending aortic wall in patients with BAV. ${ }^{6-8}$ Lastly, studies from our own group have demonstrated regional 
differences in matrix metalloproteinase production that were similarly related to BAV morphology.

With respect to the present manuscript, finding evidence of oxidative damage may provide additional clues toward identifying the underlying causes of BAV-related aortic disease. Understanding the regional distribution of ROS generation or lipid peroxidation in relation to elevated regional mechanical stress induced by aberrant blood flow may provide further insight into the role of oxidative stress and aortic remodeling. Therefore, whether these responses are handed down through heritable genetics ("hand-me-downs"), or acquired through altered mechanical stretch ("yoga pants"), these studies may indicate a need to develop therapeutic strategies to provide antioxidant therapy to patients with BAV in combination with current surgical guidelines.

\section{References}

1. Billaud M, Phillippi JA, Kotlarczyk MP, Hill JC, Ellis BW, St Croix CM, et al. Elevated oxidative stress in the aortic media of patients with bicuspid aortic valve. J Thorac Cardiovasc Surg. 2017;154:1756-62.
2. Braverman AC, Guven H, Beardslee MA, Makan M, Kates AM, Moon MR. The bicuspid aortic valve. Curr Probl Cardiol. 2005;30:470-522.

3. Michelena HI, Prakash SK, Della Corte A, Bissell MM, Anavekar N, Mathieu P, et al. Bicuspid aortic valve: identifying knowledge gaps and rising to the challenge from the International Bicuspid Aortic Valve Consortium (BAVCon). Circulation. 2014;129:2691-704

4. Birukov KG. Cyclic stretch, reactive oxygen species, and vascular remodeling Antioxid Redox Signal. 2009;11:1651-67.

5. Phillippi JA, Hill JC, Billaud M, Green BR, Kotlarczyk MP, Gleason TG. Bicuspid aortic valve morphotype correlates with regional antioxidant gene expression profiles in the proximal ascending aorta. Ann Thorac Surg. 2017;104:79-87.

6. Cotrufo M, Della Corte A, De Santo LS, Quarto C, De Feo M, Romano G, et al. Different patterns of extracellular matrix protein expression in the convexity and the concavity of the dilated aorta with bicuspid aortic valve: preliminary results. J Thorac Cardiovasc Surg. 2005;130:504-11.

7. Della Corte A, De Santo LS, Montagnani S, Quarto C, Romano G, Amarelli C et al. Spatial patterns of matrix protein expression in dilated ascending aorta with aortic regurgitation: congenital bicuspid valve versus Marfan's syndrome. $J$ Heart Valve Dis. 2006;15:20-7; discussion 27.

8. Della Corte A, Quarto C, Bancone C, Castaldo C, Di Meglio F, Nurzynska D, et al. Spatiotemporal patterns of smooth muscle cell changes in ascending aortic dilatation with bicuspid and tricuspid aortic valve stenosis: focus on cell-matrix signaling. J Thorac Cardiovasc Surg. 2008;135:8-18, e11-12.

9. Ikonomidis JS, Ruddy JM, Benton SM Jr, Arroyo J, Brinsa TA, Stroud RE, et al. Aortic dilatation with bicuspid aortic valves: cusp fusion correlates to matrix metalloproteinases and inhibitors. Ann Thorac Surg. 2012;93: 457-63. 